\title{
Modulation of Carbon Nanotubes' Perturbation to the Metabolic Activity of CYP3A4 in the Liver
}

\author{
Yi Zhang, Yabin Wang, Aijuan Liu, Sherry Li Xu, Bin Zhao, Yi Zhang, Hanfa Zou, \\ Wenyi Wang, Hao Zhu, and Bing Yan*
}

The liver plays an important role in metabolizing foreign materials, such as drugs. The high accumulation of carbon nanotubes and other hydrophobic nanoparticles in the liver has raised concerns that nanoparticles may interfere with liver metabolic function. We report here that carbon nanotubes enter hepatic cells after intravenous introduction and interact with CYP enzymes, including CYP3A4. Surface chemical modifications alter the carbon nanotubes' interactions with CYP450 enzymes in human liver microsomes. They enhance, inhibit, or have no effect on the enzymatic function of CYP3A4. Using a cheminformatics analysis, certain chemical structures are identified on the surface of the carbon nanotubes that induce an enzyme inhibitory effect or prevent disruption of CYP3A4 enzymes.

body through respiration, ingestion, or skin contact, nanoparticles can be absorbed into the human circulatory system and are then predominantly deposited into the organs of the reticuloendothelial system, such as the liver and spleen. Almost all tested nanoparticles, ${ }^{[7-19]}$ including multiwalled CNTs (MWCNTs), ${ }^{[20-23]}$ accumulate primarily in the liver regardless of administration route. Furthermore, nanoparticles have been shown to remain in the liver for an extended period of time, often months or years. ${ }^{[23,24]}$ A heavy buildup of nanoparticles in the liver causes inflammation and may adversely affect a number of liver functions, including meta-

\section{Introduction}

Cytochrome P450 (CYP450) is a large family of membranebound enzymes primarily residing in the liver. They play a key role in the metabolism of xenobiotic substances, including $75 \%$ of all marketed drugs. ${ }^{[1]}$ The perturbation of CYP450 functions seriously compromises human health and safety. ${ }^{[2]}$

In the past decade, nanotechnology has been explored for application in a variety of areas, including biomedicine. For example, carbon nanotubes (CNTs) have been investigated for applications in drug delivery, ${ }^{[3,4]}$ imaging, ${ }^{[3]}$ and tissue engineering. ${ }^{[5]}$ At the same time, more than 1826 consumer products have been marketed and are now used in our daily lives, ${ }^{[6]}$ creating multiple routes for nanoparticle exposure. After entering the human bolic functions. ${ }^{[25-27]}$ Ex vivo studies have indicated that gold, ${ }^{[28,29]}$ silver, ${ }^{[30,31]}$ and polystyrene ${ }^{[32]}$ nanoparticles inhibit CYP450 enzyme activities. Despite multiple indications that nanoparticles perturb CYP450 activities, it is unknown whether MWCNTs bind to CYP450 enzymes and perturb their activities. An even more pressing issue is whether MWCNTs' functionalization can modulate their influences on CYP450 function.

The high surface area of nanoparticles, such as MWCNTs, suggests that surface chemical modification may alter their interactions with biological systems. Combinatorial modification is a powerful approach to diversify MWCNT surface chemistries. ${ }^{[33]}$ Therefore, it is an effective way to derive novel functions from nanomaterials. We have shown that functionalized MWCNTs modified by nanocombinatorial chemistry tuned cellular functions
Dr. Y. Zhang, Y. Wang, A. Liu, Prof. B. Yan

School of Chemistry and Chemical Engineering

Shandong University

Jinan 250100, China

E-mail: drbingyan@yahoo.com

Dr. S. L. Xu, Prof. B. Zhao

State Key Laboratory of Environmental Chemistry and Ecotoxicology

Research Center for Eco-Environmental Sciences CAS

Beijing 100085, China

Dr. Y. Zhang, Prof. H. Zou

CAS Key Lab of Separation Sciences for Analytical Chemistry

National Chromatographic R\&A Center

Dalian Institute of Chemical Physics

Chinese Academy of Sciences

Dalian 116011, China
Dr. Y. Zhang

University of Chinese Academy of Sciences Beijing 100049, China

W. Wang, Prof. H. Zhu

The Rutgers Center for Computational

and Integrative Biology

Camden, NJ 08102, USA

Prof. H. Zhu

Department of Chemistry

Rutgers University

Camden, NJ 08102, USA

DOI: 10.1002/adfm.201504182 
by reprogramming the cellular signaling machinery. ${ }^{[34]}$ In this work, we investigated the interactions between surface functionalized MWCNTs and human liver microsomes. We found that (1) MWCNTs enter hepatocytes after intravenous injection in mice; (2) MWCNTs bind to a variety of CYP450 enzymes, including CYP3A4, changing the enzyme's conformation and compromising the ability of CYP3A4 to metabolize drugs, and (3) various chemical modifications of the MWCNT surface allow nanotubes to modulate CYP3A4 activity in quite different manners. Moreover, a quantitative structure-activity relationship (QSAR) study was conducted to identify the chemical features of surface ligands that should be avoided so as to not disrupt CYP3A4 activity. Our research demonstrates that the surface chemistry of MWCNTs provides a feasible way to modulate their enzyme perturbations in the liver.

\section{Results and Discussion}

\subsection{MWCNTs Enter Hepatocytes}

MWCNTs absorbed by animals largely accumulate in the liver. ${ }^{[24,35]}$ Because the CYP450 enzymes responsible for drug metabolism primarily reside in hepatocytes in the liver, ${ }^{[36]}$ we first investigated whether MWCNTs accumulated in the liver entered hepatocytes. Two hours after i.v. administration of either carboxylated MWCNT or MWCNT 1 in ICR mice, the livers were harvested, and thin sections of the liver tissues were prepared for transmission electron microscopy (TEM) analysis. TEM images showed that both types of MWCNTs accumulated in both Kupffer cells and hepatocytes (Figure 1). In Kupffer cells, a large quantity of MWCNTs was uptaken as a cluster in an endocytosis vesicle (Figure 1C). Meanwhile, fewer MWCNT clusters were found in endocytosis vesicles in hepatocytes (Figure 1D). Moreover, individual MWCNTs were able to enter the cytoplasm of hepatocytes (Figure $1 \mathrm{E}$ ) and associate with the endoplasmic reticulum membrane (Figure $1 \mathrm{~F}$ ).

The uptake of other nanoparticles (mesoporous silica nanoparticles and polystyrene nanoparticles) by hepatocytes in the liver has been observed using multiphoton fluorescence microscopy ${ }^{[37]}$ and TEM. ${ }^{[38]}$ Previous studies have also shown that well-dispersed CNTs are excreted via a biliary pathway after hepatocyte uptake. ${ }^{[39,40]}$ Hepatocytes in the liver can clear foreign particles via an endocytosis mechanism. ${ }^{[1,42]}$ Our TEM
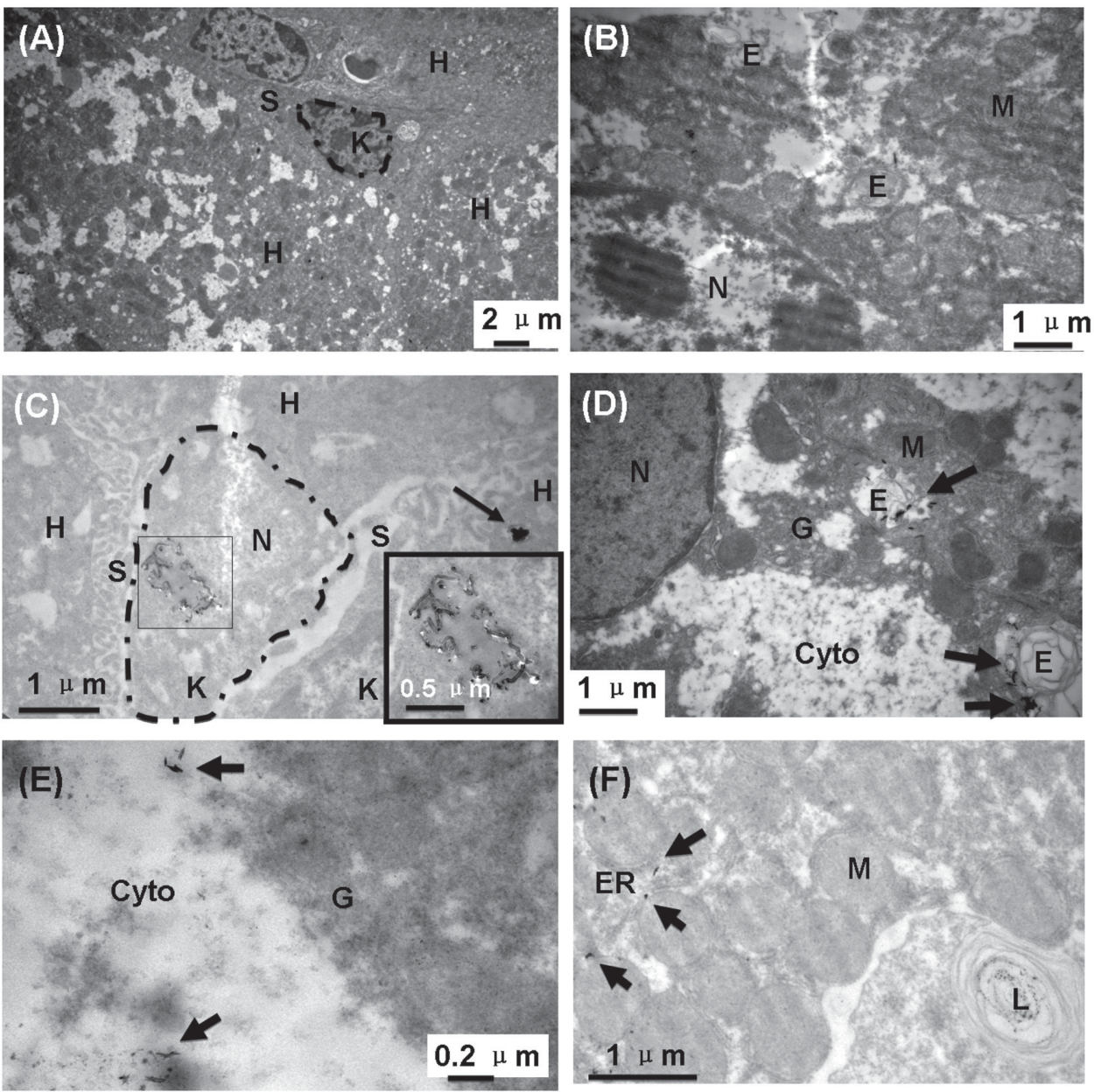

Figure 1. Internalization of MWCNTs in Kupffer cells and hepatocytes in the liver. Representative images of $A, B)$ liver slices from control and C-E) MWCNT 1 treated mice are shown. Kupffer cell in the liver is outlined by dotted lines (panels (A,C)). The dark mass in panel (C) (arrow) is glycogen granules. The MWCNTs in the Kupffer cell (panels (C)) are highlighted by a square and enlarged in the inset. The MWCNTs in the hepatocyte (panels (D-F)) are identified by red arrows. MWCNTs are found in D) endosomes, in E) the cytoplasm, and associated with the ER membrane. K, Kupffer cells; $\mathrm{H}$, hepatocytes; S, sinusoid; N, nuclear; M, mitochondria; E, endosome; Cyto, cytoplasm; ER, endoplasmic reticulum; G, Golgi apparatus; L, lysosome. 
analysis provided the first direct observation of MWCNTs in hepatocytes.

\subsection{Functionalized MWCNTs Bind CYP450 Enzymes in Human Liver Microsomes}

Because CYP450 enzymes are located in hepatocytes $^{[36]}$ and functionalized MWCNTs (f-MWCNTs) are also internalized by hepatocytes, the next part of this study investigated how MWCNTs interact with CYP450 enzymes. Surface modifications should offer MWCNTs different physiochemical properties. ${ }^{[43,44]}$ To investigate such interactions, we used human liver microsomes (HLMs) as an ex vivo system to test whether MWCNTs bind CYP450 enzymes and whether the surface chemistry of MWCNTs plays a role in such binding. In an HLM system, CYP450 enzymes are tethered to the inner membranes of mitochondria or in the endoplasmic reticulum, similar to their locations in the liver. ${ }^{[4]}$ Ten functionalized MWCNTs (f-MWCNTs) with different surface chemistries (Nos. 1-10) (Figure 5) were incubated in HLM for $30 \mathrm{~min}$. The bound enzymes were isolated and analyzed by liquid chromatography-tandem mass spectrometry (LC-MS-MS) (Table S1, Supporting Information).

As shown in Table 1, all f-MWCNTs bound CYP450 enzymes extensively, and no two f-MWCNTs had identical binding profiles. Some abundant and functionally important enzymes, such as CYP3A4 and members of the CYP2 family (CYP2C8, CYP2C9, CYP2D6 and CYP2E1) were associated with all the tested f-MWCNTs. On the other hand, other enzymes, such as CYP7B1 and CYP2J2, exhibited higher selectivity. For example, CYP7B1 bound only to MWCNT 9 but not other MWCNTs, while CYP2J2 was only bound by MWCNT 2 and 3. Because all tested f-MWCNTs have comparable physical and morphological properties (Table S2, Supporting Information), the above results indicate that the binding of CYP450 enzymes by f-MWCNTs was selective and depended only on the f-MWCNT surface chemistry.

The enzymes in each row are listed in order of their number of unique peptides. The chemical structures of each f-MWCNT are also shown.

CYP3A4 is the most abundant CYP450 enzyme (30\%- $60 \%$ of all CYP450 enzymes) ${ }^{[46]}$ and is particularly important, being responsible for the metabolism of almost half of
Table 1. Proteomic identification of $\mathrm{f}-\mathrm{MWCNT}$-bound P450 enzymes.

CYP2E1, CYP2A6, CYP2C9, CYP1A2, CYP2C8,
CYP2D6, CYP3A4, CYP4A11, CYP4F11, CYP2B6,
CYP3A5, CYP2C19, CYP3A7, CYP4V2, CYP20A1

CYP2C8, CYP2A6, CYP2C9, CYP3A4, CYP2E1, CYP1A2, CYP4A11, CYP2B6, CYP4F3, CYP2D6, CYP27A1, CYP3A5, CYP4F2, CYP8B1, CYP2J2

CYP2C8, CYP2C9, CYP3A4, CYP2A6, CYP2E1, CYP4F3, CYP4A11, CYP1A2, CYP2D6, CYP27A1, CYP2B6, CYP4F2, CYP3A5, CYP8B1, CYP2J2, CYP4F11, CYP51A1

CYP2E1, CYP2A6, CYP2C9, CYP2C8, CYP1A2 CYP4A11, CYP2D6, CYP3A4, CYP2B6, CYP3A5, CYP4F11, CYP3A7, CYP2C19, CYP20A1

CYP3A4, CYP2E1, CYP2A6, CYP2C9, CYP1A2, CYP2D6, CYP4A11, CYP4F2, CYP2C8, CYP8B1, CYP2B6, CYP27A1, CYP4F11, CYP2C19

CYP2E1, CYP2A6, CYP2C9, CYP1A2, CYP2C8, CYP4A11, CYP2D6, CYP3A4, CYP4F11, CYP2B6, CYP3A5, CYP2C19, CYP3A7, CYP4A22

CYP2E1, CYP2A6, CYP1A2, CYP4A11, CYP2C8, CYP2D6, CYP2C9, CYP3A4, CYP2B6, CYP4F11, CYP3A5, CYP2C19, CYP3A7, CYP4V2, CYP20A1

CYP3A4, CYP2E1, CYP2A6, CYP1A2, CYP2D6, CYP2C8, CYP4A11, CYP2C9, CYP2B6, CYP4F2, CYP27A1, CYP8B1, CYP4F3, CYP51A1, CYP2C19, CYP4F11, CYP4V2, CYP20A1

CYP3A4, CYP2A6, CYP4A11, CYP2E1, CYP2C9, CYP2C8, CYP1A2, CYP2D6, CYP4F2, CYP4F3, CYP2B6, CYP8B1, CYP7B1, CYP27A1

CYP2E1, CYP2A6, CYP1A2, CYP2C8, CYP2C9, CYP4A11, CYP3A4, CYP2D6, CYP4F2, CYP8B1, CYP2B6, CYP51A1, CYP27A1, CYP4F3, CYP4V2, CYP4F11 


\begin{tabular}{|c|c|c|c|c|c|c|c|c|c|}
\hline \multirow{2}{*}{$\begin{array}{c}\text { f-MWCNT } \\
\text { No. }\end{array}$} & \multicolumn{4}{|c|}{$\mathrm{HLM}\left(\mathrm{mg} \mathrm{mL}^{-1}\right)$} & \multirow{2}{*}{$\begin{array}{c}\text { f-MWCNT } \\
\text { No. }\end{array}$} & \multicolumn{4}{|c|}{ HLM (mg mL-1) } \\
\hline & 0.2 & 0.2 & 0.1 & 0 & & 0.4 & 0.2 & 0.1 & 0 \\
\hline 1 & - & $\bullet$ & & & 4 & 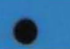 & - & & \\
\hline 5 & e & ? & 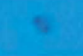 & & 6 & - & 0 & $c$ & \\
\hline 7 & c & 0 & $\theta$ & & 8 & - & e & 4 & \\
\hline 10 & ? & 6 & 6 & & & & & & \\
\hline
\end{tabular}

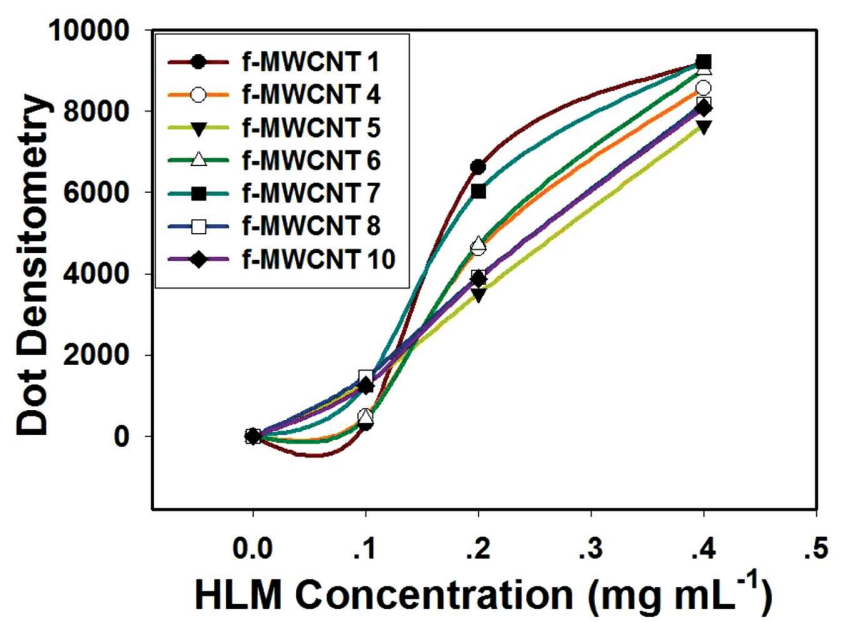

Figure 2. Bindings of $\mathrm{f}-\mathrm{MWCNTS}$ to CYP3A4 with different affinities. A) The binding affinities between f-MWCNTs and CYP3A4 were analyzed by dot blot using a CYP3A4 specific antibody and quantified by densitometry of dots. f-MWCNTs were incubated with HLM at various concentrations (from 0.1 to $0.4 \mathrm{mg} \mathrm{mL}^{-1}$ ) before blotting with the CYP3A4-specific antibody. The absolute intensity of the dots was determined by Image J. B) Dose-dependent bindings between f-MWCNTs and CYP3A4. therapeutic drugs. ${ }^{[47]}$ Our results show that CYP3A4 bound to f-MWCNTs modified by diverse surface chemistries (Table 1). To quantitatively analyze this binding, we visualized the enzyme concentration-dependent binding amounts of CYP3A4 to f-MWCNTs using a CYP3A4-specific antibody (dot blot). The binding affinity was also estimated by the densitometry of the dots. Our results show that f-MWCNTs bound to CYP3A4 in a dose-dependent manner (Figure 2A) and with different affinities. For example, MWCNTs 1 and 7 showed higher binding affinities, while MWCNT 5 had a lower affinity (Figure 2B).

As shown in Figure 2, the amount of CYP3A4 proteins bound by f-MWCNTs was almost saturated at an HLM concentration of $0.4 \mathrm{mg} \mathrm{mL}^{-1}$. However, for unsaturated concentrations (such as $0.2 \mathrm{mg} \mathrm{mL}^{-1}$ ), the CYP3A4 binding selectivity was apparent for all MWCNTs, suggesting that under competitive conditions, surface chemistry determined the binding affinity of CYP3A4 to f-MWCNTs.

\subsection{MWCNT Binding Changes CYP3A4 Conformation}

Because of the essential role of CYP3A4 in maintaining normal physiological conditions, we aimed to understand how f-MWCNTs perturb the function of CYP3A4 and how to modulate such perturbations. This is especially important when f-MWCNTs are used as carriers for the delivery of small-molecule drugs, protein therapeutics and genes..$^{[21,48]}$ Evidence of nanoparticles binding to a protein can be obtained using spectroscopic techniques, such as circular dichroism $(\mathrm{CD})^{[43]}$ and fluorescence spectroscopy. ${ }^{[4]}$ When the CYP3A4 protein was incubated
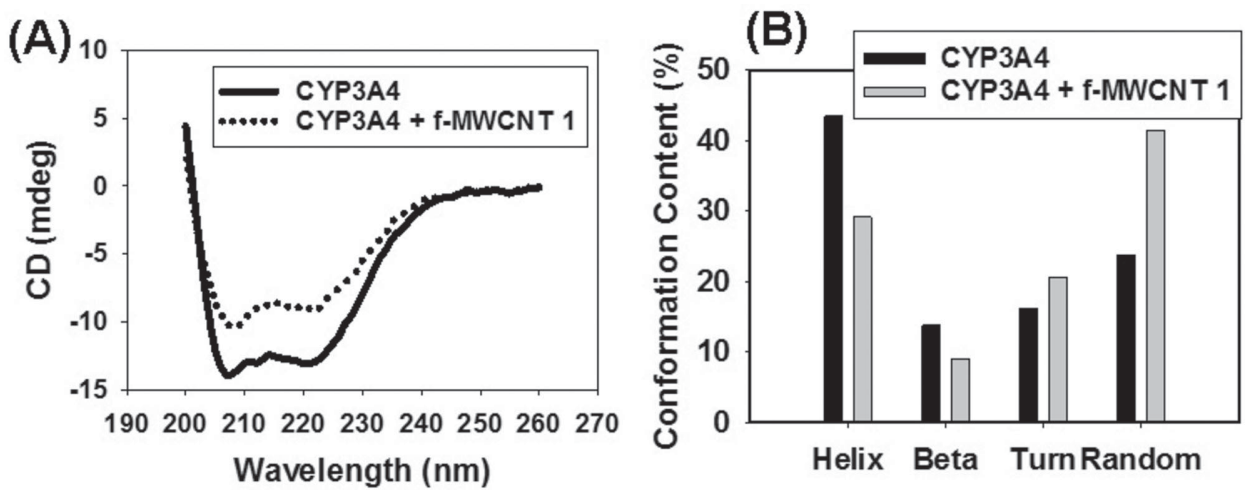

Figure 3. $f-M W C N T$ binding partially unfolds CYP3A4 protein. A) CYP3A4 exhibits a characteristic CD spectrum with negative bands at 222 and $208 \mathrm{~nm}$ and a positive band at $193 \mathrm{~nm}$. MWCNT 1 treatment decreases the ellipticity of the CD spectrum at 222 and $208 \mathrm{~nm}$. B) Content of protein secondary structures in CYP3A4 after f-MWCNT binding, as calculated by CDpro on the basis of each CD spectrum. 

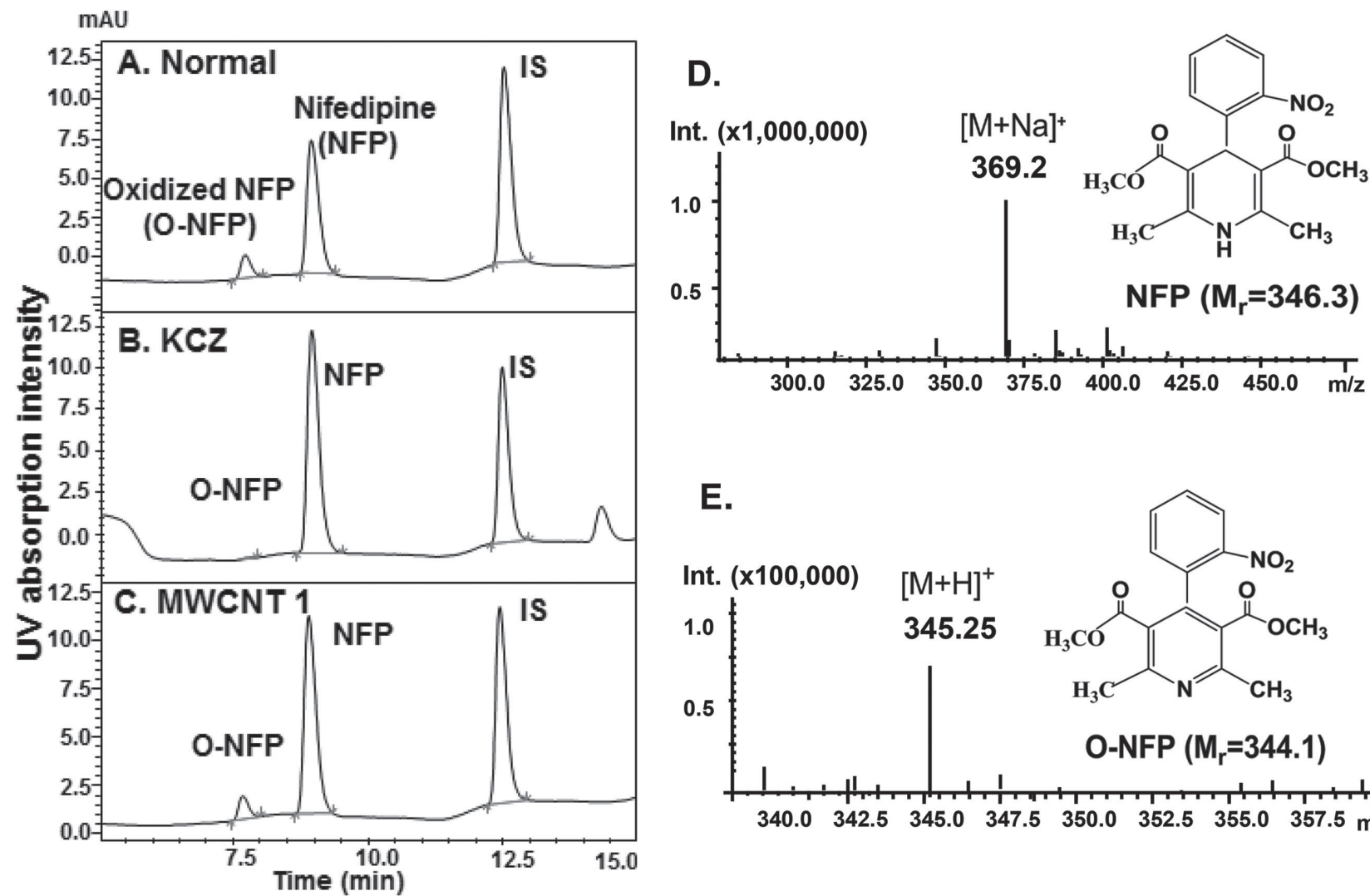

F. Relative CYP3A4 activity induced by f-MWCNT binding

\begin{tabular}{|c|c|c|c|c|c|c|c|}
\hline MWCNT No. & 1 & 4 & 5 & 6 & 7 & 8 & 10 \\
\hline $\begin{array}{c}\text { CYP3A4 } \\
\text { Activity (\%) }\end{array}$ & 72 & 100 & 90 & 125 & 124 & 125 & 94 \\
\hline
\end{tabular}

Figure 4. $\mathrm{f}-\mathrm{MWCNT}$ binding changes the metabolic activity of CYP3A4 as measured by HPLC. The HPLC chromatogram of MWCNT 1 is presented as an example of the effect of f-MWCNTs on the metabolic activity of CYP3A4. A fraction $(25 \mu \mathrm{L})$ of f-MWCNT solution (1 mg mL $\left.\mathrm{mL}^{-1}\right)$ or ketoconazole solution was incubated in HLM for $20 \mathrm{~min}$ at $37^{\circ} \mathrm{C}$ after which nifedipine was added. After 15 min, the remaining nifedipine concentration was determined by HPLC with nimodipine as an internal standard (IS). A) Incubation of HLM and the drug nifedipine. B) Same mixture as in panel (A) with the addition of ketoconazole. C) Same mixture as in panel (A) with the addition of MWCNT 1. Mass spectra of D) the parent drug nifedipine (D) and E) its metabolite. Summary of the relative CYP3A4 activity induced by F) f-MWCNT binding. For the HPLC assay, all reaction systems were $125 \mu \mathrm{L}$. Final concentrations: MWCNT 1, $0.2 \mathrm{mg} \mathrm{mL}^{-1}$; ketoconazole (KCZ), $1.3 \mu \mathrm{g} \mathrm{mL}^{-1}$; HLM, $0.5 \mathrm{mg} \mathrm{mL}^{-1}$; nifedipine, $6.9 \mu \mathrm{g} \mathrm{mL} \mathrm{L}^{-1}$; nimodipine, $0.5 \mu \mathrm{g} \mathrm{mL} \mathrm{L}^{-1}$.

with MWCNT 1, their interaction induced a large reduction in CD intensity at 208 and $222 \mathrm{~nm}$, which is typical for $\alpha$-helix structures (Figure 3). This result indicates that the binding of MWCNT 1 to CYP3A4 altered the secondary structure of the enzyme. The altered protein structural domain might be responsible for the enzymatic function; thus, this alteration may explain the reduction in CYP3A4 enzymatic activity.

The above results provide compelling evidence that f-MWCNTs bound to CYP enzymes and thereby altered the enzyme's conformation. The affinity of f-MWCNTs to CYP3A4 was governed by the physicochemical nature of the f-MWCNTs: specifically, the chemical properties of the
MWCNT surface. CNTs were reported to also bind cell membrane lipids. ${ }^{[50,51]}$ Based on these studies, we cannot exclude the possibility of non-specific interactions between CNTs and lipid molecules and such interactions might perturb the activity of CYP3A4 indirectly. However, our data showed that perturbations of f-MWCNTs on CYP3A4 were surface chemistry-dependent, indicating that direct CNT-CYP450 bindings played a major role. The binding of f-MWCNTs to CYP450 enzymes raised the question of whether f-MWCNTs affected the metabolic functions of these enzymes. We therefore tested whether f-MWCNT binding changed the enzymatic activity of CYP3A4. 

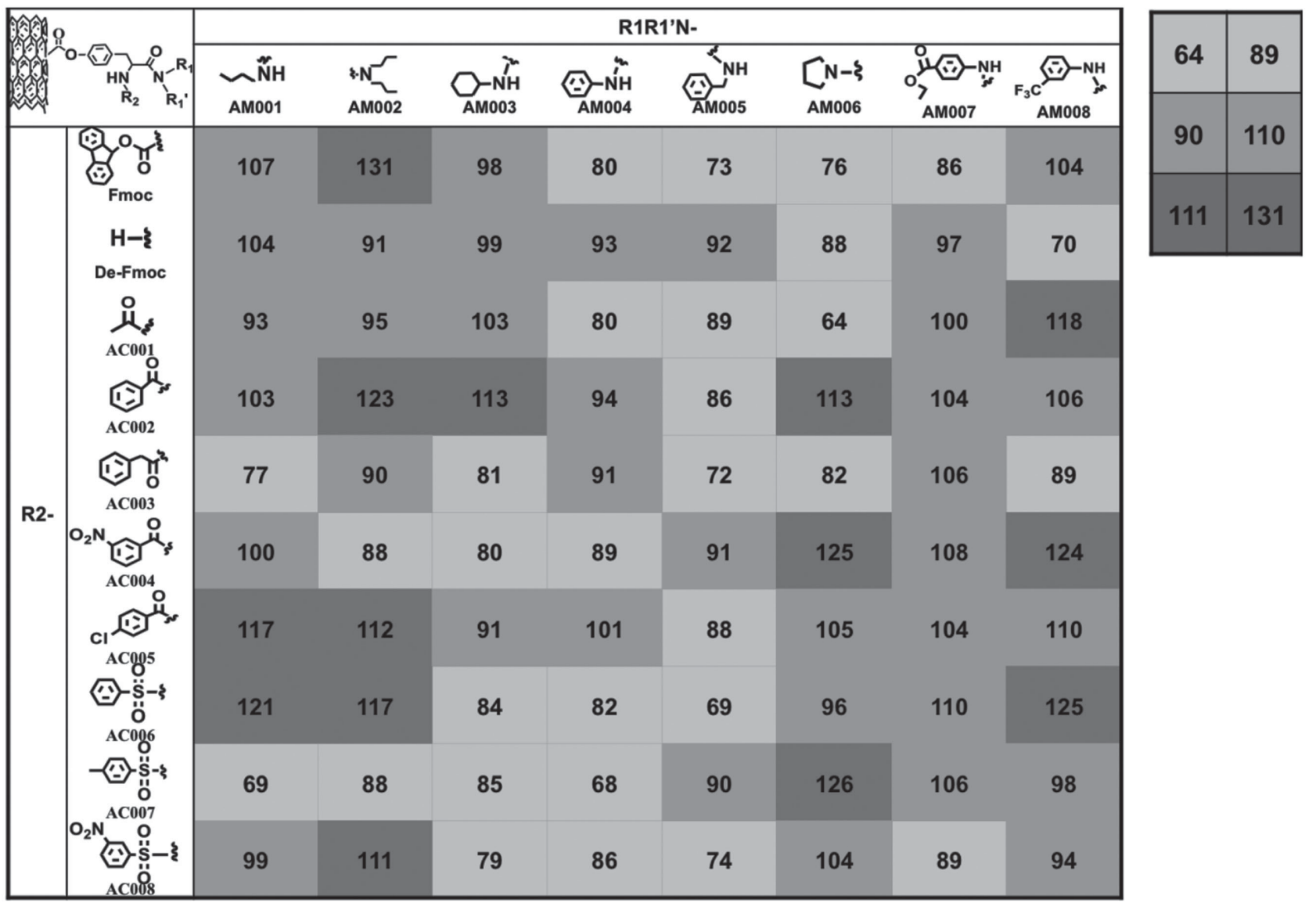

Figure 5. CYP3A4 activity is modulated by f-MWCNTs from a combinatorial MWCNT library. The CYP3A4 activity in the HLM-only group was defined as $100 \%$, and that in the ketoconazole group was defined as $0 \%$. The activity of CYP3A4 in f-MWCNT-treated groups was calculated according to the following equation: f-MWCNT's effect on CYP3A4 activity = (peak area of NFP in ketoconazole group - peak area of NFP in f-MWCNT group)/(peak area of NFP in ketoconazole group - peak area of NFP in HLM-only group).

\subsection{MWCNT Binding Changes the Enzymatic Activity of CYP3A4}

One of CYP3A4's key functions is to metabolize a drug called nifedipine by oxidation reactions. The oxidation of nifedipine by CYP3A4 can be inhibited by ketoconazole. By determining the concentrations of the remaining parent drug or the oxidized nifedipine using LC-MS, the enzymatic activity of CYP3A4 can be assessed (see Experimental Section). We then evaluated the effects of seven f-MWCNTs on the activity of CYP3A4 by analyzing the amount of nifedipine metabolized by CYP3A4 relative to an internal standards nimodipine in the absence or the presence of f-MWCNTs. Inhibition of CYP3A4 activity was determined by defining enzyme activity without MWCNT as $100 \%$ and that with CYP3A4 inhibitor ketoconazole treatment as $0 \%$. As shown in Figure 4, f-MWCNTs enhanced, suppressed, or caused no effect on the CYP3A4 activity, depending on the surface chemistry of the MWCNTs. These results demonstrated that the surface chemistry of f-MWCNTs did play a dominant role in regulating the enzymatic activity of CYP3A4 in HLM. For such studies, there is always doubt whether ligands on MWCNT surface might be metabolized by liver enzymes. Detailed knowledge on such reactions is currently lacking.
However, since f-MWCNTs caused different effects on CYP3A4 activities, it was not likely that surface ligands on MWCNTs were metabolized under our experimental conditions.

\subsection{MWCNT Surface Chemistry Affects the Functional Regulation of CYP3A4}

Systematic modification of the surface chemistry of MWCNTs was accomplished by the synthesis of a nanocombinatorial MWCNT library. ${ }^{[33]}$ To test the effects of this combinatorial f-MWCNT library, which contains 80 members, on CYP3A4 activity in a high-throughput format, we determined the remaining parent drug concentration after incubating library members with NFP in HLM. As shown in Figure 5, multiple f-MWCNTs with diverse chemistries modulated the enzymatic activity of CYP3A4 over a broad range (60\%-131\%). There are 32 out of 80 MWCNTs inhibited CYP3A4 activity (activity $<90 \%$ ), and 14 out of $80 \mathrm{MWCNTs}$ enhanced CYP3A4 activity (activity $>110 \%$ ). Noticeably, 34 f-MWCNTs did not perturb the activity of CYP3A4 (activity between $90 \%$ and $110 \%$ ).

Whether through their purposeful introduction for biomedical applications or via accidental exposure from polluted 
Table 2. Analysis of important descriptors.

\begin{tabular}{lcccc}
\hline$P$-value & Active & Inactive & Descriptor & Description \\
\hline $35.51 \%$ & 0.64 & 0.99 & F10 O-F & Frequency of O-F at topological distance 10 \\
$28.85 \%$ & 1.31 & 2.02 & F02 C-N & Frequency of C-N at topological distance 2 \\
$14.86 \%$ & 0.86 & 1.30 & F05 N-O & Frequency of N-O at topological distance 5
\end{tabular}

environment, it is desirable that these materials do not perturb CYP3A4 function. The CYP3A4 inert f-MWCNTs discovered in this work may have a great potential for biomedicine and other applications.

Although some small molecules deactivate CYP3A4 enzyme, ${ }^{[52]}$ others can also activate this enzyme. ${ }^{[53]}$ It has been suggested that, like small molecules, nanoparticle binding may also enhance CYP450 activity. ${ }^{[28]}$ To our knowledge, the findings presented herein provide the first experimental evidence to support this hypothesis. Enhanced CYP3A4 enzyme activity may lead to low therapeutic efficacy ${ }^{[52]}$ or drug intoxication, as has been observed in the case of enzymatic inhibition. ${ }^{[54]}$ Thus, the enhancement of CYP3A4 activity by f-MWCNTs is as toxic as enzyme inhibition and should be identified and avoided.

The systematic modification of f-MWCNTs modulated their interactions with CYP3A4. This finding raised the question of how the f-MWCNT surface chemistry was correlated to the perturbations in CYP3A4 activity. To answer this question, we conducted a QSAR study, which has recently been used to predict the physiochemical properties and cytotoxicity of specific nanoparticles. ${ }^{[55,56]}$ First, according to the activity readouts, we classified MWCNTs in the library into three groups: the enhancement group (activity $>110 \%, 14$ MWCNTs), the neutral group (activity from 90 to $110 \%, 34$ MWCNTs) and the inhibition group (activity <90\%, 32 MWCNTs). Because the amount of data available for the MWCNT enhancement group was insufficient, only QSAR analyses based on a combination of the inhibition (activity defined as 1) and neutral (activity defined as 0) groups was conducted using molecular descriptors calculated by Dragon ver. 6.0. In total, 503 chemical descriptors were generated for the surface molecules attached to the MWCNTs.

The $k$ nearest neighbor $(k N N)$ is a QSAR modeling approach used to classify unknown compounds based on the class membership of its $k$ nearest neighbor compounds in chemical space. ${ }^{[57,58]}$ Based on the Dragon chemical descriptors, $100 \mathrm{kNN}$ models were built with a fivefold cross validation and a combined accuracy of $78.77 \%$. After the $k \mathrm{NN}$ modeling, the above descriptors were prioritized in terms of the frequency with which they appeared among all $100 \mathrm{kNN}$ models,

Table 3. Structure alerts for CYP3A4 inhibition.

\begin{tabular}{|c|c|c|c|c|}
\hline Alter & Alter-containing molecule No. & Actives & Avg. activity & Statistical significance [\%] \\
\hline & 17 & 12 & 85.82 & 95.3 \\
\hline & 9 & 8 & 81.33 & 98.2 \\
\hline & 7 & 6 & 83.14 & 94.5 \\
\hline & 5 & 3 & 89.6 & 68.8 \\
\hline & 4 & 3 & 81.75 & 75 \\
\hline
\end{tabular}



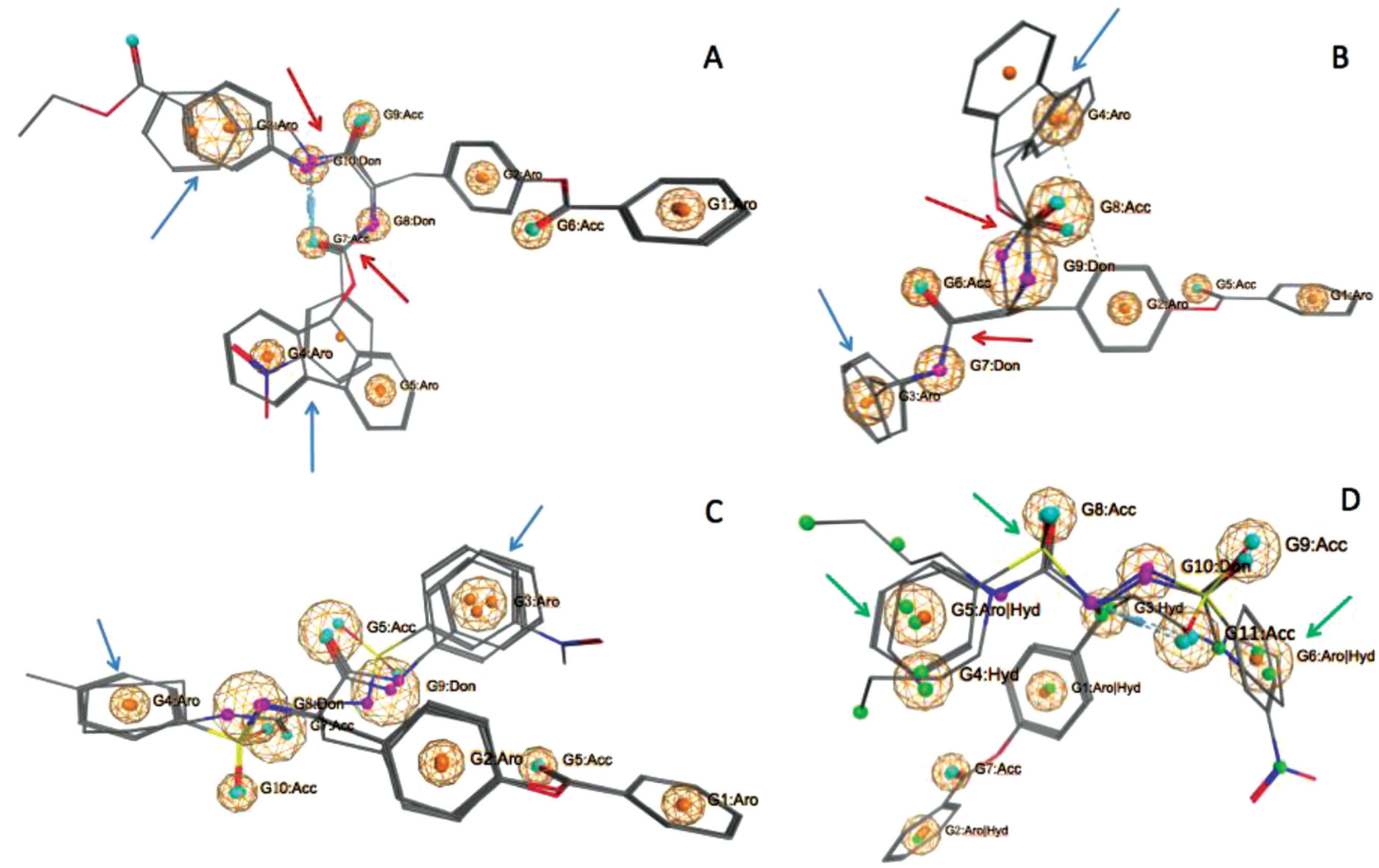

Figure 6. Identification of pharmacophores on MWCNT surface molecules. A-C) The structures of three groups of surface molecules on MWCNTs that show similar inhibitory effects toward CYP3A4 activity used for inhibitory pharmacophore search. Blue arrows indicate the benzene/aromatic rings on both ends of the side chain, and red arrows indicate the two - CONR2 groups. D) The identification of pharmacophores in parts of the surface molecules on MWCNTs that do not change CYP3A4 activity. Green arrows indicate reduced aromaticity and fewer tertiary nitrogens in specific locations. Hyd: hydrophobic centroid (green dots); Acc: hydrogen bond acceptor (blue dots); Don: hydrogen bond donor (purple dots); Aro: aromatic ring (orange dots).

and the 20 highest descriptors were chosen for this analysis. The 20-descriptor-reduced pool was submitted to a $t$-test, and the three topologically based descriptors with $P$ value $<40 \%$ are shown in Table 2. Based on their $P$ values, these descriptors are considered to differ significantly between active and inactive MWCNTs (Table 2).

Next, the Case Ultra program (version 1.5) was employed to analyze the relationships between f-MWCNTs and the corresponding CYP3A4 activities. The modeling identified five functional group alerts that significantly inhibited CYP3A4 activity (Table 3). We then conducted a pharmacophore search using f-MWCNT surface molecules with key Dragon descriptors that overlapped with those identified in the Case Ultra Modeling. Our study shows that to minimize the inhibition of CYP3A4 activity, long and complex side chains and aromatic rings should be avoided (Figure 6A-C). Moreover, fewer $-\mathrm{NH}_{2}$ and more $-\mathrm{CONR}_{2}$ also lead to CYP3A4 binding and should be avoided (Table 3). Meanwhile, the pharmacophore analysis, which was based on a mapping of the surface molecules of the MWCNTs not affecting CYP3A4, provides additional support for the results of the above analysis. The results of the pharmacophore analysis indicate that fewer aromatic rings and fewer tertiary nitrogens in specific positions reduce the effect of MWCNTs on CYP3A4 (Figure 6D).

\section{Conclusion}

Extensive nanoparticle deposition in the liver is common for all nanomaterials, regardless of whether exposure is a direct consequence of the use of nanomaterials for biomedical applications or accidental exposures to nanoparticles in the environment. The accumulation of nanoparticles in the liver causes undesirable perturbations to metabolic functions, including CYP enzymatic functions. In this investigation, we have demonstrated that f-MWCNTs perturb the enzymatic activity of CYP3A4 by binding to the protein and altering its conformation. We have shown that the chemical modification of MWCNT surfaces can modulate f-MWCNT perturbations of CYP3A4 activity in the liver. Based on a QSAR analysis, we found that long and complex hydrophobic or aromatic side chains on the nanotube surfaces are responsible for inducing the inhibitory effects of f-MWCNTs. We also discovered that the pharmacophores with lower aromaticity and fewer tertiary nitrogen atoms are more likely to generate safe MWCNTs. Our findings provide key insights into the understanding of the relationships between physicochemical features of f-MWCNTs and regulation of the CYP3A4 function in the liver. This information will be helpful in guiding the rational design of safe MWCNTs for applications in biomedicine and consumer products. 


\section{Experimental Section}

Reagents and Antibodies: Nifedipine (N7634), nimodipine (N149), and ketoconazole (K1003) were purchased from Sigma-Aldrich (St Louis, MO, USA). The CYP3A4 antibody was purchased from Cell Signaling (Boston, MA, USA). Horseradish peroxidase conjugated secondary antibody was purchased from BioRad (Richmond, CA, USA). Nitrocellulose membranes and luminescence reagents were purchased from EMD Millipore Corporation. HLMs and NADPH Regenerating System (Solution A and Solution B) were commercially available from BD Biosciences. Human cytochrome P450 3A4 (H5042) was purchased from Sigma-Aldrich. All reagents for HPLC were purchased from Thermo Fisher Scientific.

Functionalized MWCNT Preparation: The synthesis methods for f-MWCNTs in the combinatorial library have been reported in detai in our previous study. ${ }^{[33]}$ For animal experiments, the suspensions of f-MWCNTs were prepared in PBS with 1\% Tween 80 and sonicated for $1 \mathrm{~h}$ to facilitate dispersion.

Animal Experiments: The animals used in this study were 5 week old male ICR mice (VitalRiver Corp., Beijing) with a body weight of 20-25 g. All materials used for animal experiments were sterilized. f-MWCNTs were injected into the caudal vein at a concentration of $15 \mathrm{mg} \mathrm{kg}^{-1}$ body weight. Two hours after injection, animals were sacrificed, and the liver was fixed for super-thin section preparation.

Transmission Electron Microscopy: For electron microscopy, tissue specimens were fixed overnight in glutaraldehyde $(2.5 \%)$ in PBS $(0.1 \mathrm{M}$, $\mathrm{pH}$ 7.4). After washing three times in PBS, the tissues were fixed for $1 \mathrm{~h}$ in $2 \% \mathrm{OsO}_{4}$ solution. Next, tissues were washed three times in $\mathrm{ddH}_{2} \mathrm{O}$ and dehydrated stepwise in $\mathrm{EtOH}$. Tissues were polymerized with Spurr resin at $68{ }^{\circ} \mathrm{C}$ for $15 \mathrm{~h}$. The embedded specimens were subsequently thin-sectioned into $70 \mathrm{~nm}$ slices and viewed on a Hitachi H-7650 TEM operating at $80 \mathrm{kV}$.

LC-MS-MS for Protein Identification: The Protein was dissolved in denaturing buffer $(\mathrm{pH} \mathrm{7.4)}$ containing $8 \mathrm{~m}$ urea and $50 \mathrm{~mm}$ Tris- $\mathrm{HCl}$. The protein was reduced by adding DTT $(2 \mu \mathrm{M})$ and alkylated by adding iodoacetamide (IAA) (4 $\mu \mathrm{m})$. After that, the solution was ultrafiltered and then digested by trypsin and desalted with a C18 SPE column. The resulting peptides were analyzed using the LTQ-OrbiTrap Velos (Thermo Fisher Scientific Inc., San Jose, CA). The data analysis was completed by searching with MaxQuant (version 1.1.1.36).

Circular Dichroism Analysis: Human cytochrome P450 3A4 $\left(26 \mu \mathrm{g} \mathrm{mL}^{-1}\right)$ was incubated with an $\mathrm{f}-\mathrm{MWCNT}$ suspension $\left(70 \mu \mathrm{g} \mathrm{mL}^{-1}\right)$ in PBS for $30 \mathrm{~min}$ before circular dichroism (J-810, JASCO, Japan) analysis. The conformations of CYP3A4 with and without f-MWCNTs were recorded at room temperature. The secondary structure content of CYP3A4 was calculated using CDPro software.

Dot Blot: Different concentrations $\left(0.4,0.2,0.1\right.$, and $\left.0 \mathrm{mg} \mathrm{mL}^{-1}\right)$ of HLM in PBS were incubated with f-MWCNTs (final concentration, $0.18 \mathrm{mg} \mathrm{mL}^{-1}$ ) for $30 \mathrm{~min}$ at $37^{\circ} \mathrm{C}$. The mixture was then centrifuged at $15000 \mathrm{rpm} \mathrm{min} \mathrm{m}^{-1}$ for $10 \mathrm{~min}$. The f-MWCNT sediment was washed five times with PBS and resuspended in PBS $(100 \mu \mathrm{L})$, of which $1 \mu \mathrm{L}$ was blotted to form the dot on the nitrocellulose (NC) membrane. The blots were blocked with $5 \%$ milk for $1 \mathrm{~h}$ and then incubated with the CYP3A4-specific antibody for $1 \mathrm{~h}$. After washing three times with PBS containing 0.5\% Tween 20, the blots were incubated in the secondary antibody for an additional hour. After washing again, the blots were immersed in the chemiluminescent solution for visualization on a $2600 \mathrm{C}$ automatic X-ray film processor. The gray level of the dots was semiquantified by Imagel software.

Nifedipine Metabolism in HLM: First, HLM $\left(20 \mathrm{mg} \mathrm{mL}^{-1}, 3.13 \mu \mathrm{L}\right)$ was diluted in PBS $(89 \mu \mathrm{L})$. This HLM solution was mixed with f-MWCNT dispersion $\left(25 \mu \mathrm{L}, 1 \mathrm{mg} \mathrm{mL} \mathrm{m}^{-1}\right)$ in tubes and blended at $800 \mathrm{rpm}$ $\mathrm{min}^{-1}$ for $20 \mathrm{~min}$ at $37^{\circ} \mathrm{C}$. Next, nifedipine $\left(2.5 \mu \mathrm{L}, 10 \times 10^{-3} \mathrm{M}\right)$ and NADPH preparation $(7.5 \mu \mathrm{L}, \mathrm{A}: \mathrm{B}=5: 1)$ were added; the reaction was allowed to continue for $15 \mathrm{~min}$ at $37{ }^{\circ} \mathrm{C}$. The reaction was quenched by immersing the tubes in ice. Next, the internal standard nimodipine solution $\left(125 \mu \mathrm{L}, 5 \mu \mathrm{g} \mathrm{mL} \mathrm{m}^{-1}\right)$ was added dropwise while the above mixture was gently vortexed. The new solution was then centrifuged at $15000 \mathrm{rpm} \mathrm{min}{ }^{-1}$ for $10 \mathrm{~min}$. The quantity of nifedipine in the supernatant was measured by high performance liquid chromatography (HPLC-MS 2010EV, Shimadzu, Japan). The HPLC column was packed with octadecyl silane chemically bonded silica. The flowing mobile phase was composed of methanol and water with the following gradient elution: 50:50 for $\min 0-4,65: 35$ for $\min 5-8,75: 25$ for $\min 9-12$, and 50:50 for $\min 13$ ). The flow rate was $0.3 \mathrm{~mL} \mathrm{~min}^{-1}$, and the absorbance of the target was detected at $238 \mathrm{~nm}$.

Quantitative Structure-Activity Relationship Analysis: Based on the enzymatic activity of CYP3A4, f-MWCNTs were classified into inhibition and neutral groups. kNN modeling and Case Ultra modeling were then applied to analyze the functional group features of MWCNTs selected from each group. For kNN modeling, 503 Dragon descriptors were generated, and the top 20 important descriptors were identified based on the occurrence frequency among all qualifying kNN models. Case Ultra models were built to analyze the relationships between f-MWCNTs and their corresponding CYP3A4 activities. The conclusions of the QSAR study were based on the overlap between important Dragon descriptors and the structures identified in the Case Ultra models. Finally, the pharmacophores were identified to emphasize important features.

\section{Supporting Information}

Supporting Information is available from the Wiley Online Library or from the author.

\section{Acknowledgements}

Y.Z. and Y.W. contributed equally to this work. The authors thank Dr. Lei Yang for technical assistance in the early phase of this project. This work was supported by the Natural Science Foundation of China (21137002, 91543204 and 21525730), the Strategic Priority Research Program of the Chinese Academy of Sciences (XDB14030400 and XDB14030401), the NIEHS of the NIH (R15ES023148), and the ColgatePalmolive Grant for Alternative Research.

Received: September 30, 2015

Revised: November 13, 2015

Published online: December 21, 2015

[1] F. P. Guengerich, Chem. Res. Toxicol. 2007, 21, 70.

[2] M. Pirmohamed, B. K. Park, Toxicology 2003, 192, 23.

[3] M. Martincic, G. Tobias, Expert Opin. Drug Delivery 2015, 12, 563.

[4] S. Dhar, Z. Liu, J. Thomale, H. Dai, S. J. Lippard, J. Am. Chem. Soc. 2008, 130, 11467

[5] B. S. Harrison, A. Atala, Biomaterials 2007, 28, 344.

[6] Consumer Products Inventory, http://www.nanotechproject.org/ cpi/products/ (accessed: September 2015).

[7] T. Skajaa, D. P. Cormode, P. A. Jarzyna, A. Delshad, C. Blachford, A. Barazza, E. A. Fisher, R. E. Gordon, Z. A. Fayad, W. J. M. Mulder, Biomaterials 2011, 32, 206.

[8] C. L. Zavaleta, K. B. Hartman, Z. Miao, M. L. James, P. Kempen, A. S. Thakor, C. H. Nielsen, R. Sinclair, Z. Cheng, S. S. Gambhir, Small 2011, 7, 2232.

[9] W.-S. Cho, M. Cho, J. Jeong, M. Choi, H.-Y. Cho, B. S. Han, S. H. Kim, H. O. Kim, Y. T. Lim, B. H. Chung, J. Jeong, Toxicol. Appl. Pharmacol. 2009, 236, 16.

[10] Z. Liu, K. Chen, C. Davis, S. Sherlock, Q. Cao, X. Chen, H. Dai, Cancer Res. 2008, 68, 6652.

[11] P. Jani, G. W. Halbert, J. Langridge, A. T. Florence, J. Pharm. Pharmacol. 1990, 42, 821.

[12] J. X. Wang, G. Q. Zhou, C. Y. Chen, H. W. Yu, T. C. Wang, Y. M. Ma, G. Jia, Y. X. Gao, B. Li, J. Sun, Y. F. Li, F. Jiao, Y. L. Zhao, Z. F. Chai, Toxicol. Lett. 2007, 168, 176. 
[13] M. Zhu, W. Feng, Y. Wang, B. Wang, M. Wang, H. Ouyang, Y. Zhao, Z. Chai, Toxicol. Sci. 2009, 107, 342

[14] L. Wang, L. Wang, W. Ding, F. Zhang, J. Nanosci. Nanotechnology 2010, 10, 8617.

[15] F. Leuschner, P. Dutta, R. Gorbatov, T. I. Novobrantseva, J. S. Donahoe, G. Courties, K. M. Lee, J. I. Kim, J. F. Markmann, B. Marinelli, P. Panizzi, W. W. Lee, Y. Iwamoto, S. Milstein, H. Epstein-Barash, W. Cantley, J. Wong, V. Cortez-Retamozo, A. Newton, K. Love, P. Libby, M. J. Pittet, F. K. Swirski, V. Koteliansky, R. Langer, R. Weissleder, D. G. Anderson, M. Nahrendorf, Nat. Biotechnol. 2011, 29, 1005.

[16] G. S. Kang, P. A. Gillespie, A. Gunnison, A. L. Moreira, K. TchouWong, L. Chen, Environ. Health Perspect. 2011, 119, 176.

[17] K. Loeschner, N. Hadrup, K. Qvortrup, A. Larsen, X. Gao, U. Vogel, A. Mortensen, H. Lam, E. Larsen, Part. Fibre Toxicol. 2011, 8, 18.

[18] N. V. Gopee, D. W. Roberts, P. Webb, C. R. Cozart, P. H. Siitonen, J. R. Latendresse, A. R. Warbitton, W. Y. William, V. L. Colvin, N. J. Walker, Toxicol. Sci. 2009, 111, 37.

[19] T. H. Umbreit, S. Francke-Carroll, J. L. Weaver, T. J. Miller, P. L. Goering, N. Sadrieh, M. E. Stratmeyer, J. Appl. Toxicol. 2012, $32,350$.

[20] H. Ali-Boucetta, K. Kostarelos, Adv. Drug Delivery Rev. 2013, 65, 2111.

[21] G. Liang, L. Yin, J. Zhang, R. Liu, T. Zhang, B. Ye, Y. Pu, J. Toxicol. Environ. Health, Part A 2010, 73, 463.

[22] L. Lacerda, H. Ali-Boucetta, M. A. Herrero, G. Pastorin, A. Bianco, M. Prato, K. Kostarelos, Nanomedicine 2008, 3, 149.

[23] X. Deng, G. Jia, H. Wang, H. Sun, X. Wang, S. Yang, T. Wang, Y. Liu, Carbon 2007, 45, 1419.

[24] G. Qu, Y. Bai, Y. Zhang, Q. Jia, W. Zhang, B. Yan, Carbon 2009, 47, 2060.

[25] M. B. Esch, G. J. Mahler, T. Stokol, M. L. Shuler, Lab Chip 2014, 14, 3081

[26] J. Zongfei, Z. Danying, L. Ling, S. Xizhong, D. Xiaoyong, D. Ling, W. Minhong, L. Yuanfang, Nanotechnology 2009, 20, 445101.

[27] Z. Danying, D. Xiaoyong, J. Zongfei, S. Xizhong, D. Ling, W. Minghong, G. Taoying, L. Yuanfang, Nanotechnology 2010, 21, 175101.

[28] M. Ye, L. Tang, M. Luo, J. Zhou, B. Guo, Y. Liu, B. Chen, Nanoscale Res. Lett. 2014, 9, 642.

[29] A. Sereemaspun, P. Hongpiticharoen, R. Rojanathanes, P. Maneewattanapinyo, S. Ekgasit, W. Warisnoicharoen, Int. J. Pharmcol. 2008, 4, 492.

[30] K. Kulthong, R. Maniratanachote, Y. Kobayashi, T. Fukami, T. Yokoi, Xenobiotica 2012, 42, 854.

[31] J. G. Lamb, L. B. Hathaway, M. A. Munger, J. L. Raucy, M. R. Franklin, Drug Metab. Dispos. 2010, 38, 2246.

[32] E. Fröhlich, T. Kueznik, C. Samberger, E. Roblegg, C. Wrighton, T. R. Pieber, Toxicol. Appl. Pharmcol. 2010, 242, 326.

[33] H. Zhou, Q. Mu, N. Gao, A. Liu, Y. Xing, S. Gao, Q. Zhang, G. Qu, Y. Chen, G. Liu, Nano Lett. 2008, 8, 859.

[34] Y. Zhang, L. Wu, C. Jiang, B. Yan, Chem. Res. Toxicol. 2015, 28, 296.
[35] N. Gao, Q. Zhang, Q. Mu, Y. Bai, L. Li, H. Zhou, E. R. Butch, T. B. Powell, S. E. Snyder, G. Jiang, B. Yan, ACS Nano 2011, 5, 4581.

[36] J. Loeper, V. Descatoire, M. Maurice, P. Beaune, J. Belghiti, D. Houssin, F. Ballet, G. Feldmann, F. Guengerich, D. Pessayre, Gastroenterology 1993, 104, 203.

[37] S.-H. Cheng, F.-C. Li, J. S. Souris, C.-S. Yang, F.-G. Tseng, H.-S. Lee, C.-T. Chen, C.-Y. Dong, L.-W. Lo, ACS Nano 2012, 6, 4122.

[38] S. R. Popielarski, S. Hu-Lieskovan, S. W. French, T. J. Triche, M. E. Davis, Bioconjugate Chem. 2005, 16, 1071.

[39] Z. Liu, C. Davis, W. Cai, L. He, X. Chen, H. Dai, Proc. Natl. Acad. Sci. USA 2008, 105, 1410

[40] S. Y. Hong, G. Tobias, K. T. Al-Jamal, B. Ballesteros, H. Ali-Boucetta, S. Lozano-Perez, P. D. Nellist, R. B. Sim, C. Finucane, S. J. Mather, M. L. H. Green, K. Kostarelos, B. G. Davis, Nat. Mater. 2010, 9, 485.

[41] E. Kuntz, H.-D. Kuntz, Hepatology Principles and Practice: History, Morphology Biochemistry, Diagnostics Clinic, Therapy, Vol. 3, Springer, Berlin 2006.

[42] P. J. Lawrence, PhD Dissertation, State University of New York at Stony Brook, Stony Brook, NY, USA 2007

[43] Q. Mu, W. Liu, Y. Xing, H. Zhou, Z. Li, Y. Zhang, L. Ji, F. Wang, Z. Si, B. Zhang, B. Yan, J. Phys. Chem. C 2008, 112, 3300.

[44] Q. Mu, G. Jiang, L. Chen, H. Zhou, D. Fourches, A. Tropsha, B. Yan, Chem. Rev. 2014, 114, 7740.

[45] T. Gram, Methods Enzymol. 1974, 31, 225.

[46] D. J. Waxman, Arch. Biochem. Biophys. 1999, 369, 11.

[47] C. E. Fluck, P. E. Mullis, A. V. Pandey, Biochem. Biophys. Res. Commun. 2010, 401, 149.

[48] A. Bianco, K. Kostarelos, M. Prato, Curr. Opin. Chem. Biol. 2005, 9, 674.

[49] C. Rocker, M. Potzl, F. Zhang, W. J. Parak, G. U. Nienhaus, Nat. Nanotechnol. 2009, 4, 577.

[50] M. Gasser, P. Wick, M. Clift, F. Blank, L. Diener, B. Yan, P. Gehr, H. F. Krug, B. Rothen-Rutishauser, Part. Fibre Toxicol. 2012, 9, 17.

[51] P. Yi, K. L. Chen, Environ. Sci. Technol. 2013, 47, 5711.

[52] L. Goodman, L. Limbird, P. Milnoff, A. Gilman, J. Hardman, Goodman Q G Gilman's: The Pharmacological Basis of Therapeutics, McGraw-Hill, New York 1996.

[53] K. Mizuno, M. Katoh, H. Okumura, N. Nakagawa, T. Negishi, T. Hashizume, M. Nakajima, T. Yokoi, Drug Metab. Dispos. 2009, 37, 345.

[54] J. Cheng, X. Ma, K. W. Krausz, J. R. Idle, F. J. Gonzalez, Drug Metab. Dispos. 2009, 37, 1611.

[55] A. A. Toropov, J. Leszczynski, Chem. Phys. Lett. 2006, 433, 125.

[56] T. Puzyn, B. Rasulev, A. Gajewicz, X. Hu, T. P. Dasari, A. Michalkova, H.-M. Hwang, A. Toropov, D. Leszczynska, J. Leszczynski, Nat. Nanotechnol. 2011, 6, 175.

[57] N. S. Altman, Am. Stat. 1992, 46, 175

[58] G. W. Kauffman, P. C. Jurs, J. Chem. Inf. Comput. Sci. 2001, 41, 1553. 